\title{
Deterministic aggregation kinetics of superparamagnetic colloidal particles
}

Colin P. Reynolds, Kira E. Klop, François A. Lavergne, Sarah M. Morrow, Dirk G. A. L. Aarts, and Roel P. A. Dullens

Citation: The Journal of Chemical Physics 143, 214903 (2015); doi: 10.1063/1.4936323

View online: http://dx.doi.org/10.1063/1.4936323

View Table of Contents: http://aip.scitation.org/toc/jcp/143/21

Published by the American Institute of Physics

\section{Articles you may be interested in}

Aggregation kinetics of paramagnetic colloidal particles

The Journal of Chemical Physics 102, 5492 (1998); 10.1063/1.469278

Relating Brownian motion to diffusion with superparamagnetic colloids American Journal of Physics 85, 265 (2017); 10.1119/1.4975382

Communication: Radial distribution functions in a two-dimensional binary colloidal hard sphere system The Journal of Chemical Physics 140, 161106 (2014); 10.1063/1.4872365

Two-stage kinetics of field-induced aggregation of medium-sized magnetic nanoparticles The Journal of Chemical Physics 146, 114902 (2017); 10.1063/1.4977993

Bifurcations and chaos of a vibration isolation system with magneto-rheological damper AIP Advances 6, 035310 (2016); 10.1063/1.4944556

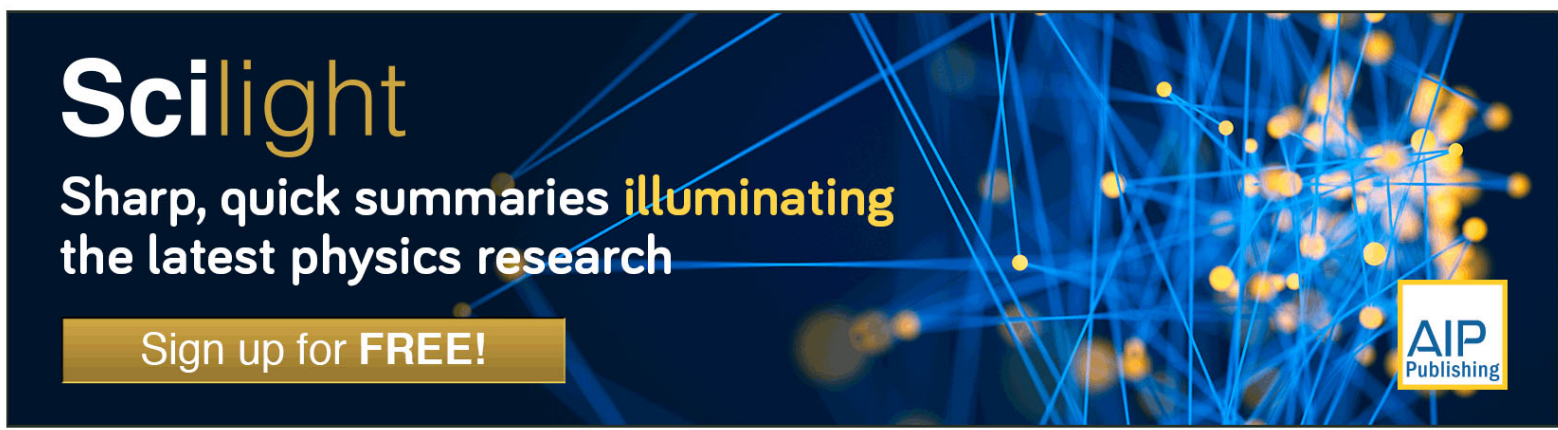




\title{
Deterministic aggregation kinetics of superparamagnetic colloidal particles
}

\author{
Colin P. Reynolds, Kira E. Klop, François A. Lavergne, Sarah M. Morrow, \\ Dirk G. A. L. Aarts, and Roel P. A. Dullens \\ Department of Chemistry, Physical and Theoretical Chemistry Laboratory, South Parks Road, \\ Oxford OX1 3QZ, United Kingdom
}

(Received 2 September 2015; accepted 11 November 2015; published online 1 December 2015)

\begin{abstract}
We study the irreversible aggregation kinetics of superparamagnetic colloidal particles in two dimensions in the presence of an in-plane magnetic field at low packing fractions. Optical microscopy and image analysis techniques are used to follow the aggregation process and in particular study the packing fraction and field dependence of the mean cluster size. We compare these to the theoretically predicted scalings for diffusion limited and deterministic aggregation. It is shown that the aggregation kinetics for our experimental system is consistent with a deterministic mechanism, which thus shows that the contribution of diffusion is negligible. () 2015 AIP Publishing LLC. [http://dx.doi.org/10.1063/1.4936323]
\end{abstract}

\section{INTRODUCTION}

Externally imposed magnetic and electric fields have long been known to induce structural changes in suspensions of colloidal particles. ${ }^{1,2}$ For magnetically responsive systems in particular, there have been several experimental realizations of structure formation, including magnetic holes in ferrofluid carriers ${ }^{2}$ and magnetic particles in aqueous solvents. ${ }^{3,4}$ This structure formation can produce large changes in the physical and optical properties of the magnetorheological fluid. ${ }^{5,6}$ Practical applications include civil engineering solutions for seismic shock absorbers and bridge stabilizers, advanced prosthetics, and it finds use in the auto-motives industry. 7,8

The formation of chains and clusters is responsible for the observed changes in physical parameters, such as the viscosity. ${ }^{6}$ The precise mechanism of aggregation affects the dynamics of chain formation and thus the response profile of a device based on these materials. ${ }^{9}$ Investigations have therefore focused on cluster formation at low particle packing fractions with work in two and three dimensions, ${ }^{3,4}$ real space experiments, ${ }^{10-21}$ scattering experiments, ${ }^{22-29}$ and simulations. ${ }^{30-34}$

Theoretical predictions based on a diffusion limited irreversible aggregation mechanism, ${ }^{35,36}$ along with experimental ${ }^{3,4,22,29}$ and simulation work, ${ }^{37,38}$ point to the mean cluster size, $\langle L\rangle$, growing as a power law in time, $t$, with an exponent $z^{\prime}:\langle L\rangle \sim t^{z^{\prime}}$. Predictions of these exponents have been given in the range of $0.5<z^{\prime}<0.6 .{ }^{3}$ Many investigations are reported in terms of a mean weighted by the number of particles in a cluster, $\langle S\rangle$, that grows with a different exponent $z:\langle S\rangle \sim t^{z}$, though it has been established that $z \approx z^{\prime}{ }^{10,11,17}$ In addition, it is proposed that time can be rescaled to account for the dependence of the average cluster size on the external magnetic field, $B$, and the packing fraction, $\phi .3,4,10,22,30,39$

When magnetic interactions dominate Brownian forces, a deterministically controlled aggregation process is proposed. ${ }^{3,17,18}$ In this regime, it has been predicted that the growth of the mean cluster size follows a power law, like in the diffusion limited case, but with a different packing fraction and magnetic field dependence. ${ }^{12,32,33,40}$ However, a detailed and quantitative experimental study of deterministic aggregation that simultaneously considers both the packing fraction and field dependence is lacking. In this article, we use a superparamagnetic colloidal model to study the field induced aggregation using video-microscopy. In particular, we measure the growth of the mean cluster size and propose a method for explicitly extracting the parameters that characterise the field and packing fraction dependence.

The paper is organised as follows: we will start with Sec. II, which briefly introduces the interactions of magnetic dipoles and the theories of diffusion limited aggregation and deterministically controlled aggregation. The experimental system is then introduced in Sec. III with details of the particles, microscope, and fields used. The data treatment is detailed and used to extract the scaling parameters for the packing fraction and field dependence. The results of this processing are presented in Sec. IV for a range of packing fractions and field strengths. We discuss the results in light of the two proposed theories with particular focus on the packing fraction and field dependence.

\section{THEORY}

\section{A. Interactions between magnetic particles}

The interaction between superparamagnetic colloidal particles in the presence of an external field is dominated by their magnetic moments. This interaction is often characterised by the dimensionless interaction parameter $\lambda$, which is the interaction energy between two dipolar particles $i$ and $j$ at contact and aligned parallel to the magnetic field, relative to the thermal energy $k_{\mathrm{B}} T$ ( $k_{\mathrm{B}}$ is Boltzmann's constant and $T$ the temperature). ${ }^{41}$ The interaction energy, $U_{i j}$, is given by

$$
\frac{U_{i j}}{k_{\mathrm{B}} T}=\frac{\lambda}{2(r / 2 a)^{3}}\left(1-3 \cos ^{2} \theta\right) \text {. }
$$


Here $a, r$, and $\theta$ are the particle radius, the inter-particle separation, and the angle between the bond-vector connecting the particles $i$ and $j$ and the direction of the applied magnetic field, respectively. The interaction parameter is given by

$$
\lambda=\frac{\pi a^{3} \chi^{2} B^{2}}{9 k_{\mathrm{B}} T \mu_{0}},
$$

where $\mu_{0}$ is the permeability of free space, $\chi$ is the dimensionless volume susceptibility of the particles, and $B$ the strength of the magnetic field. Note that we neglect mutual induction effects. For $\lambda \gg 1$, the aggregation process becomes essentially irreversible, ${ }^{41,42}$ with thermal energy insufficient to break particles apart. The result is a distribution of different cluster sizes, $s$, where $s$ is the number of particles in a cluster. The mean is defined as

$$
\langle L\rangle=\frac{\sum_{s} s n_{s}}{\sum_{s} n_{s}},
$$

where $n_{s}$ is the number of cluster of size $s$ present in a snapshot. ${ }^{17}$ The number weighted mean cluster size is given by

$$
\langle S\rangle=\frac{\sum_{s} s^{2} n_{s}}{\sum_{s} s n_{s}} .
$$

\section{B. Diffusion limited aggregation}

Diffusion limited aggregation kinetics in dipolar systems has been described extensively, see, e.g., Refs. 22, 38, 39, and 43 , and therefore, we only briefly recap the essentials. From Smoluchowski's kinetic equation of aggregation, one can, in certain limits, give expressions for the average cluster size as a function of time for an irreversible diffusive limited aggregation mechanism. ${ }^{36}$ In essence, the equation is a rate equation involving a reaction kernel that describes the rate at which clusters of size $i$ and $j$ join. Taking the collision cross section of clusters of different sizes to be the same and the diffusion coefficient of a cluster to have a power law dependence on its size, $D_{s} \sim s^{\gamma}$, the average cluster size grows as $^{3}$

$$
\langle L\rangle \sim t^{1 /(1-\gamma)} .
$$

Hence, if the friction of a chain is taken to be proportional to its length, then $\gamma=-1$ and $\langle L\rangle \sim t^{1 / 2}{ }^{36}$ The choice of $\gamma$ gives a range of values for $0.5<z^{\prime}<0.6 .^{1,3,4}$ For the diffusion dominated case a time rescaling based on the time to diffuse to a capture radius, within which particles are rapidly drawn together, i.e., the typical time for doublet formation, $t_{\mathrm{DIFF}}^{*}$, is proposed. ${ }^{3,15,23}$ This capture radius is often defined by the region around the particle in which the interaction due to the magnetic dipoles equals the thermal energy, $U_{i j} / k_{\mathrm{B}} T=1$. In $2 \mathrm{D}$ and for a given packing fraction $\phi$, this leads to the following expression for $t_{\mathrm{DIFF}}^{*} \cdot 3,15,30$

$$
t_{\mathrm{DIFF}}^{*} \sim \frac{a^{2}}{D \lambda^{2 / 3} \phi} .
$$

This implies that for diffusion limited aggregation, noting the definition of $\lambda$ (Eq. (2)), this characteristic time is proportional to $1 / B^{4 / 3}$ and $1 / \phi$.

\section{Deterministic aggregation}

Next, we introduce the deterministically controlled aggregation largely following Ref. 40 with a potential of the form of Eq. (1). For small clusters of size $s$ that are relatively far apart and aligned with the magnetic field, the interaction energy between the clusters is approximated by $s^{2}$ multiplied by the inter-particle potential $U_{i j}$, Eq. (1), with $\theta=0^{\circ}$,

$$
\frac{U_{s}}{k_{\mathrm{B}} T}=-\frac{s^{2} \lambda}{(r / 2 a)^{3}} .
$$

The force, $F_{s}=-\partial U_{s} / \partial r$, is then given by

$$
F_{s}=-\frac{24 a^{3} s^{2} \lambda k_{\mathrm{B}} T}{r^{4}}
$$

This attractive magnetic force is balanced by a drag force, $F_{\text {drag }}$, which depends on the cluster size $s .{ }^{31}$ Here, we take the friction of a chain to be proportional to the number of particles it contains, so that $F_{\mathrm{drag}}=-s 6 \pi \eta a v,{ }^{36}$ where $\eta$ is the viscosity of the solvent and $v$ the velocity of the cluster. In the overdamped limit, the equation of motion becomes

$$
F_{s}+F_{\mathrm{drag}}=-\frac{24 a^{3} s^{2} \lambda k_{\mathrm{B}} T}{r^{4}}-6 s \pi \eta a \frac{\mathrm{d} r}{\mathrm{~d} t}=0 .
$$

We integrate the equation of motion taking the final separation as zero and the initial separation, $R_{0}$, between clusters of size $s$ in a system of packing fraction $\phi$ as $R_{0}=a(s / \phi)^{1 / 2}$. It then directly follows that the time for two clusters of size $s$ to come together, $\tau_{s}$, is

$$
\tau_{s}=\left(\frac{\pi \eta a^{3} s^{3 / 2}}{20 \lambda \phi^{5 / 2} k_{B} T}\right) .
$$

Assuming hierarchical growth, the total time for a cluster of size $s$ to form, $t_{s}$, is then given by the sum $t_{s}=\tau_{s / 2}+\tau_{s / 4}$ $+\cdots+\tau_{1}$. The expression for $t_{s}$ corresponds to a geometric series, $t_{s}=\tau_{s}\left(\epsilon+\epsilon^{2}+\cdots\right)$, with $\epsilon=(1 / 2)^{3 / 2}$. In this model, the cluster size $s$ is equal to the mean cluster size $\langle L\rangle$, which is therefore found to grow as

$$
\langle L\rangle \sim\left(\frac{20 k_{B} T \lambda \phi^{5 / 2}}{\pi \eta a^{3}} t\right)^{2 / 3},
$$

predicting $z^{\prime}=2 / 3 .^{40}$ A typical time scale, $t_{\mathrm{DET}}^{*}$, can then be identified using that $\langle L\rangle \sim\left(t / t_{\mathrm{DET}}^{*}\right)^{2 / 3}$, so that

$$
t_{\mathrm{DET}}^{*} \sim \frac{a^{2}}{D \lambda \phi^{5 / 2}},
$$

which is proportional to $1 / B^{2}$ and $1 / \phi^{5 / 2}$.

\section{EXPERIMENTAL METHODS}

\section{A. Colloidal model system}

We use aqueous suspensions of superparamagnetic polystyrene spheres (carboxylic acid functionalised Dynabeads ${ }^{\circledR}$ M-270, Invitrogen), with a diameter of $3.0 \mu \mathrm{m}$ and a mass density of $\rho=1.6 \mathrm{~g} \mathrm{~cm}^{-3}$. Due to the magnetic nanoparticles embedded in the polymer matrix, these particles are magnetic and the magnitude of the magnetic moment 
is proportional to the external field for sufficiently small fields. Using SQUID (superconducting quantum interference device) measurements, we confirmed that our experiments are conducted within this region of reversible linear magnetisation and find a dimensionless volume susceptibility of $\chi=0.76$.

The particles are contained in a $200 \mu \mathrm{m}$ thick quartz glass cell (Hellma) and the packing fraction $\phi$ is varied between 0.01 and 0.05 . Due to their relatively high mass density, they form a mono-layer at the bottom of the sample cell. The out-of-plane fluctuations are negligible as can be inferred from the particle's gravitational height,

$$
h_{g}=\frac{3 k_{\mathrm{B}} T}{4 \pi a^{3} \Delta \rho g},
$$

with $\Delta \rho$ the mass density difference between the solvent and the particle, and $g$ the acceleration due to gravity. For our system, $h_{g}=0.06 \mu \mathrm{m}$, which is only a fraction of the particle radius. The Brownian time, which is the time it takes for a particle to diffuse over a distance equal to its diameter, is given by

$$
t_{b}=\frac{6 \pi \eta a^{3}}{k_{\mathrm{B}} T}
$$

and is $\sim 20$ s when these particles are suspended in a $20 \%$ ethanol solution.

\section{B. Magnetic field}

The external magnetic field was provided by two 470 turn solenoids, placed symmetrically either sides of the sample cell giving a stable, locally uniform, magnetic field. The magnetic field was varied in the range of $0-3.2 \mathrm{mT}$, as was characterised with a Gaussmeter (Hirst Magnetic Instruments Ltd. GM07 Gaussmeter, PT002 Hall Probe). This corresponds to an interaction parameter between $0 \leq \lambda \leq 1000$.

\section{Video microscopy and image analysis}

An Olympus CK41 inverted microscope equipped with a Ximea xiQ USB3 camera is used to acquire $2048 \times 20488$ bit grey-scale images. The field of view is $1150 \times 1150 \mu \mathrm{m}^{2}$. To capture the aggregation process properly, the acquisition rate is adjusted during the experiment so that frames are exponentially distributed in time giving us a uniform sampling on a logarithmic time axis. From the microscopy images, the particle positions are detected by a very simple thresholding approach using the fact that the particles have a bright spot at their centres (see inset in Fig. 1).

To identify the particles that belong to the same cluster, we note that there is a dark region around all the particles that is picked up by the thresholding (see inset in Fig. 1). We consider that when coordinates are encapsulated by the same dark region they are members of the same cluster. If a dark region is in contact with the edge of the images, we discard the coordinates it encapsulates as we cannot know the true size of that cluster. This process of establishing clusters has an advantage over using a simple distance criterion in that if a chain incorporates a single anomalous particle that is not detected we are not considering this as two separate chains.

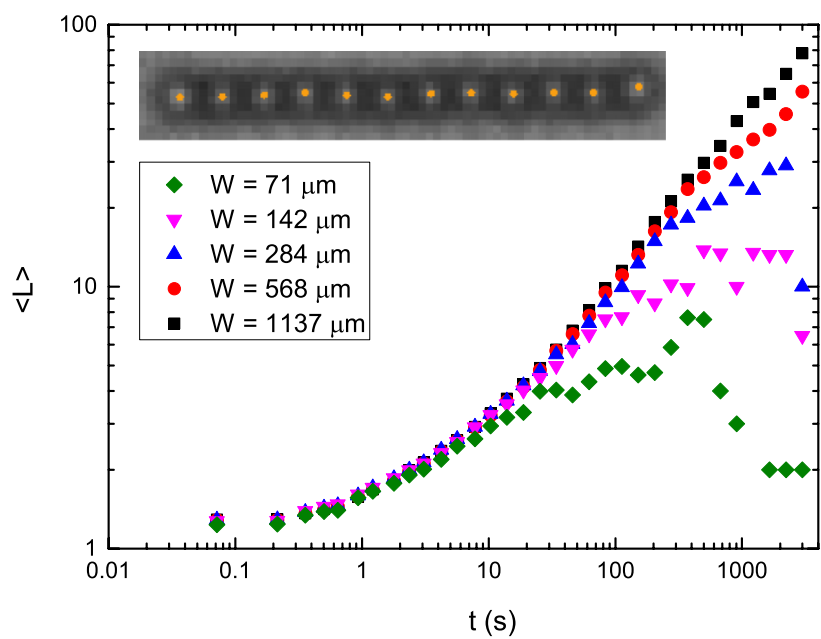

FIG. 1. The mean cluster size $\langle L\rangle$, Eq. (3), against time for different observable rectangular regions of width $\mathrm{W}$ for a single experimental run with a packing fraction $\phi=0.037$ and an external magnetic field $B=3.12 \mathrm{mT}$. The full field of view is $1150 \times 1150 \mu \mathrm{m}^{2}$. The inset shows a microscopy images of a typical cluster with the results of our image analysis algorithm plotted over it.

Once the clusters have been identified, the mean cluster size $\langle L\rangle$ (Eq. (3)) is computed. As the typical cluster length becomes on the order of the image width, $W$, finite size artefacts can start to impede on the analysis of the cluster sizes. To test these effects, we systematically increased $\mathrm{W}$ at the maximum magnetic field and highest packing fraction used, $B=3.12 \mathrm{mT}$ and $\phi=0.037$, respectively, and monitored the convergence of the mean cluster size as $W$ increases (Fig. 1). Note that this analysis is performed on a single experimental run, so the error in $\langle L\rangle$ cannot be defined. This analysis shows that only at very late times the mean cluster size may be affected by the finite field of view.

\section{RESULTS AND DISCUSSION}

Figure 2 presents a series of images capturing the process of aggregation into linear chains upon application of an external magnetic field. Particles, starting from an initially random configuration, come together to form chains of one particle thick. Chains of two particles thick are negligible at the low packing fractions used as they are not formed in significant quantities over the time scale of observation. Inherent to the strong interactions, the chains are rigid and thermal fluctuations are small. As shown in the inset in Fig. 2(a), which shows the $x$-positions of a small collection of particles over the first $40 \mathrm{~s}$, the particles follow well-defined trajectories which are reminiscent of deterministic rather than diffusive motion. We observed no break up events; when clusters joined to form a larger cluster, they did so irreversibly.

The mean cluster size $\langle L\rangle$, Eq. (3), as a function of time is presented in Fig. 3(a) for different magnetic fields at a constant packing fraction. A clear monotonic growth of cluster size with time can be seen with faster growth of clusters at higher magnetic fields. Fig. 3(b) shows the analogous graph for different packing fractions at a constant magnetic field. 

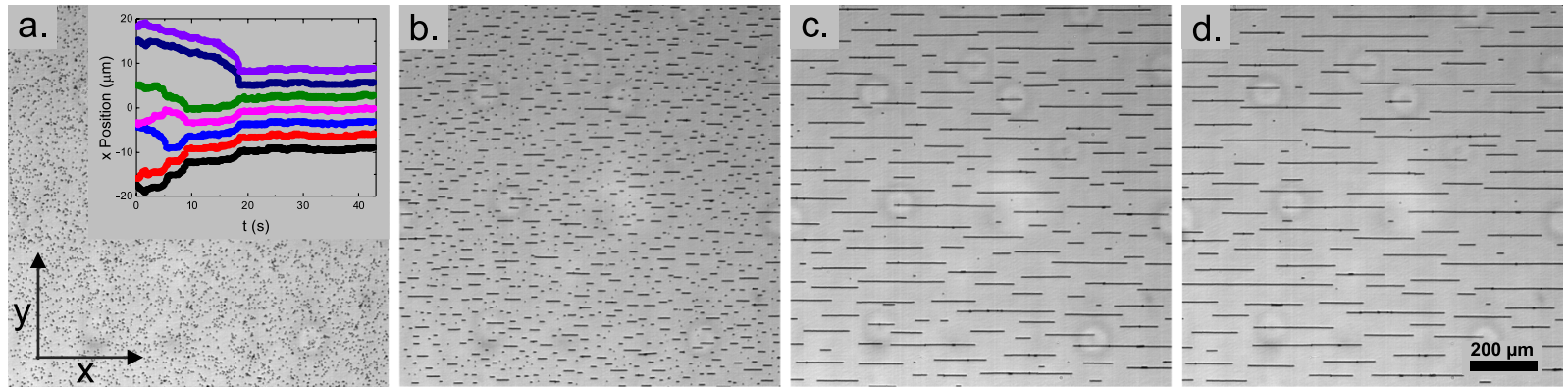

FIG. 2. Microscopy images for different times showing the magnetic field induced chain formation. Panel (a) shows the initial state of the system before the magnetic field is applied. Panels (b-d) show the system at, respectively, $95 \mathrm{~s}, 1680 \mathrm{~s}$, and $3000 \mathrm{~s}$ after a constant uniaxial magnetic field is applied in the $\mathrm{x}$ direction at $t=0 \mathrm{~s}$. The packing fraction is $\phi=0.031$ and the magnetic field is $B=1.88 \mathrm{mT}$. The inset shows the $x$-positions of a small collection of particles over the first $40 \mathrm{~s}$.

Again, a monotonic growth is observed, now with faster growth at higher packing fractions. In both cases, the long time behaviour shows an apparent power law scaling of the mean cluster size with time, consistent with earlier reports. ${ }^{10}$ We note that at early times, just after the field is applied, the cluster size depends on the packing fraction, which is due to a small number of particles that are in contact in the random starting configuration. At intermediate and long times, the effects of this initial structuring are negligible.
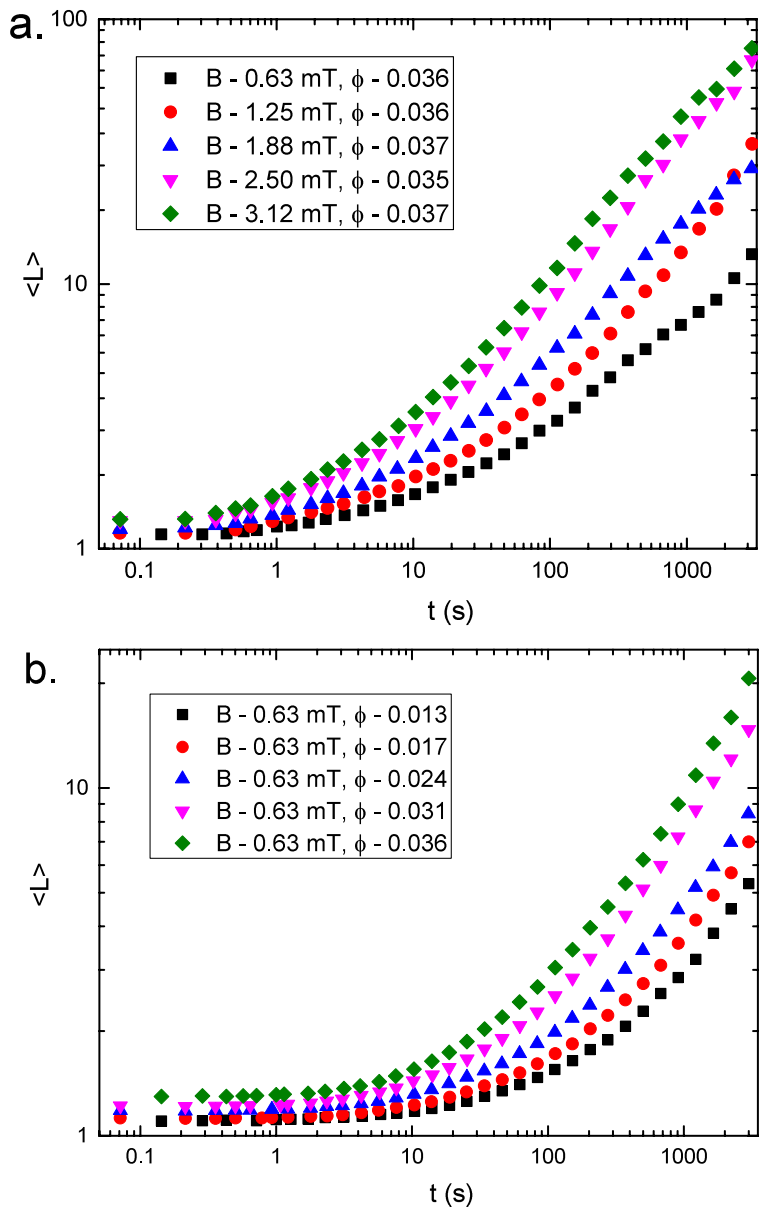

FIG. 3. The mean cluster size, $\langle L\rangle$, as a function of time, $t$, for experimental runs at (a) a constant packing fraction $\phi \approx 0.036$ and an increasing magnetic field $B$ and (b) a constant magnetic field $B=0.63 \mathrm{mT}$ and an increasing packing fraction $\phi$.
In considering the magnetic field and packing fraction dependence of the cluster size, it is common practice to plot a proposed time rescaling and judge how effective a given theory is at removing the dependence on the magnetic field and packing fraction by the quality of data collapse.,30,42,44 Another approach is to fit to a particular scaling law with the packing fraction dependence and field dependence as free fitting parameters. ${ }^{45,46}$ However, in the approach we use here, we do not require a specific functional form for the cluster growth. Instead, we write that the mean cluster size, $\langle L\rangle$, grows as a monotonic scaling function $f$ of unspecified form of the variable $t^{*}=\phi^{\alpha} B^{\beta} t$, such that

$$
\langle L\rangle=f\left(\phi^{\alpha} B^{\beta} t\right) .
$$

Here, $\alpha$ and $\beta$ are scaling constants that characterise the packing fraction and field dependence of the growing mean cluster size. Comparison to Eqs. (11) and (12) shows that the deterministic model predicts values of $\alpha=5 / 2$ and $\beta=2$, while the diffusive theory Eq. (6) predicts values of $\alpha=1$ and $\beta=4 / 3$.

Equation (15) states that a particular mean cluster size is generated by the same $\phi^{\alpha} B^{\beta} t$. As the magnetic field $B$ is

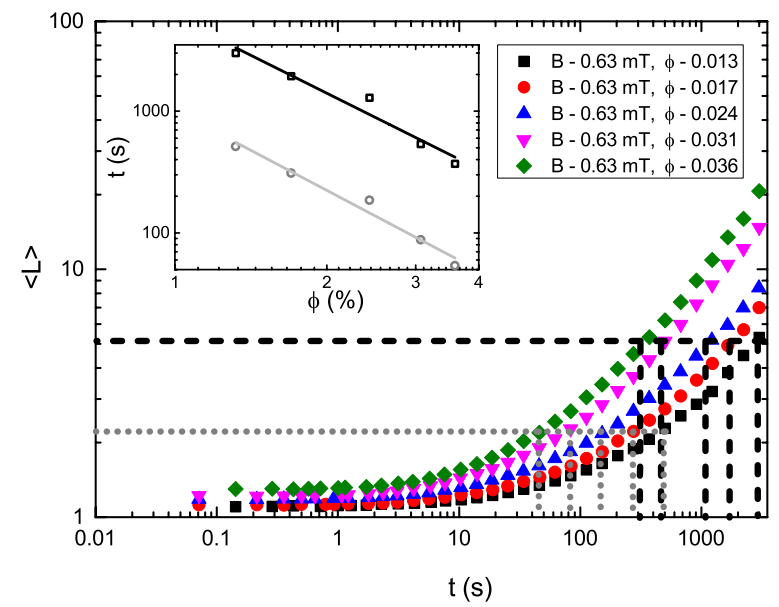

FIG. 4. The mean cluster size $\langle L\rangle$ against time for experimental runs at different packing fractions $\phi$ but with the same magnetic field $B$. The horizontal lines cut the curves at the same cluster size. The vertical lines, dropping from the intersects, show the time at which that cluster size occurred. The inset shows the packing fraction against the time at which a particular cluster size was achieved and the solid line is the linear fit to the data. 
TABLE I. A summary of the experimentally obtained values for the exponents $\phi^{\alpha}$ and $B^{\beta}$ alongside the theoretical prediction for the diffusive and deterministic aggregation.

\begin{tabular}{lccc}
\hline \hline Exponent & Experimental & Diffusive & Deterministic \\
\hline$\alpha$ & 2.44 & 1 & $5 / 2$ \\
$\beta$ & 1.61 & $4 / 3$ & 2 \\
\hline \hline
\end{tabular}

strictly controlled in our experiments, we first consider chain formation for varying $\phi$ and constant $B$. In particular, we measure the time at which a given cluster size is reached for different packing fractions $\phi$, as shown in Fig. 4. Because $\phi^{\alpha} t$ is constant for a given mean cluster size, the exponent $\alpha$ is directly obtained from the gradient of $\ln t$ vs. $\ln \phi$ as shown in the inset of Fig. 4. The determination of the exponent $\alpha$ is robust with respect to the choice of the given cluster size as is also illustrated in Fig. 4, where the light grey lines correspond to the same analysis for a smaller given cluster size. In general, we choose the largest mean cluster size common to all data sets as the given cluster size.

Having established the value of $\alpha$, an analogous procedure is performed to determine the exponent $\beta$ for the magnetic
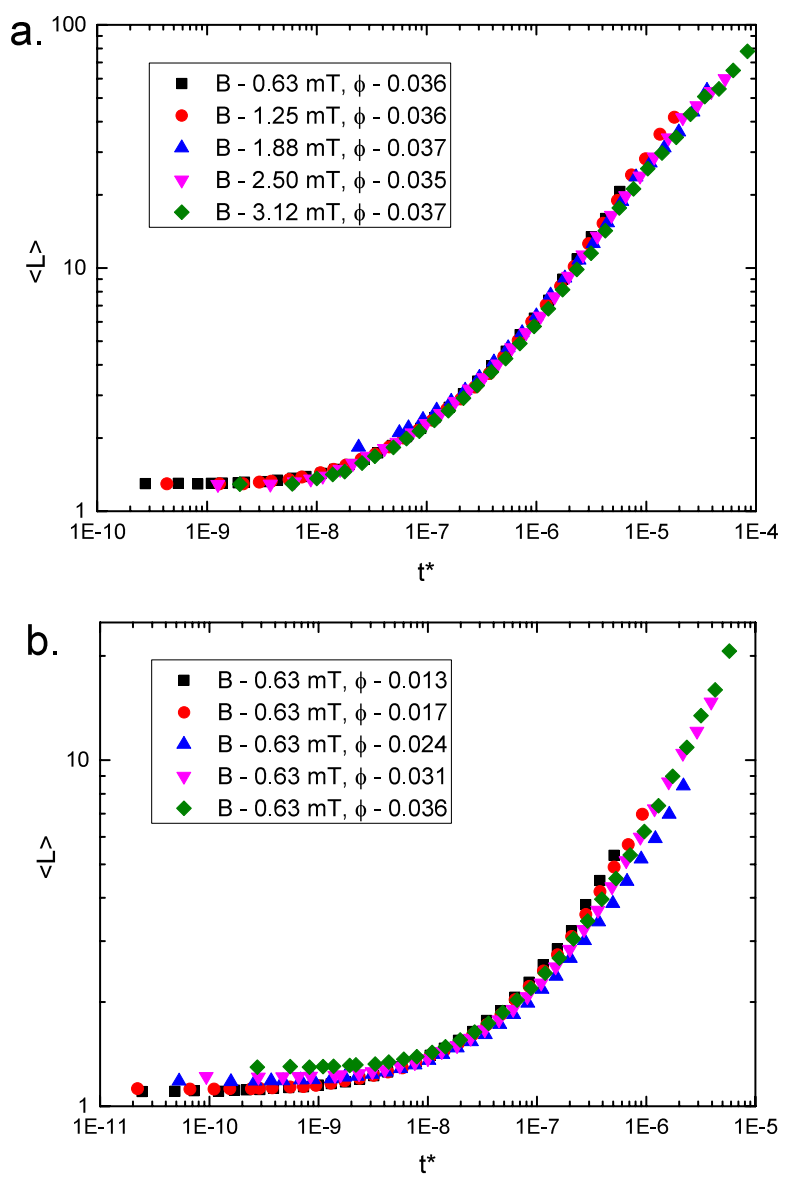

FIG. 5. The mean cluster size, $\langle L\rangle$, as a function of the rescaled time, $t^{*}=\phi^{\alpha} B^{\beta} t$, where $\alpha$ and $\beta$ are the values obtained from the experiments (see Table I). In panel (a), the experimental runs at a constant packing fraction, $\phi \approx 0.036$, and an increasing magnetic field $B$ are presented, while panel (b) shows the experimental runs a constant magnetic field, $B=0.63 \mathrm{mT}$, and an increasing packing fraction $\phi$. Note that the data presented here are the same as in Fig. 3. field dependence. The mean cluster size can be considered as $\langle L\rangle=f\left(B^{\beta}\left(\phi^{\alpha} t\right)\right)$ and because the factor $\phi^{\alpha} t$ is known, the effect of the magnetic field strength on the growth kinetics can be determined. To this end, we determine the $\phi^{\alpha} t$ at which a given mean cluster size is reached for different field strengths $B$. The exponent $\beta$ is then extracted from a linear fit of $\ln \left(\phi^{\alpha} t\right)$ vs. $\ln B$.

The values for the exponents $\alpha$ and $\beta$ extracted from our experiments, alongside the values as predicted from diffusive and deterministic aggregations, are summarised in Table I. We also determined the exponents using the number weighted mean $\langle S\rangle$, Eq. (4), and very similar values were obtained, which shows that both means scale in the same way with $\phi$ and $B$. The value for $\alpha$ is consistent with deterministic aggregation, while the exponent $\beta$ is in between what is expected for diffusive and deterministic aggregations. We note however that a very small fraction of particles $(\leq 0.1 \%)$ is pinned to the glass surface, which will hinder the growth of clusters, particularly at long times as they are incorporated into chains. As such, this may contribute to the deviation from theory in our experimentally measured $\beta$ value. Nevertheless, the combination of the excellent agreement of the packing fraction dependence and the field dependence being larger than the diffusive prediction makes us confident that deterministic aggregation is the key mechanism behind the chain formation in our system.

The data presented in Fig. 3 can now be re-plotted as a function of the rescaled time $t^{*}=\phi^{\alpha} B^{\beta} t$ using the experimentally determined values for $\alpha$ and $\beta$ (Table I). The rescaled plots are presented in Fig. 5 and as expected, an excellent data collapse is observed for the magnetic field (a) and packing fraction (b) dependent data. Finally, we show in Fig. 6 the master curve of $\langle L\rangle$ versus $t^{*}$ averaged over all experimental runs. The data are excellently described by a power law fit of the form, $\langle L\rangle=a+b t^{* z^{\prime}}$. Here, $a$ represents the zero time cluster size, which we expect to be close to unity as at the low packing fractions used most particles will not

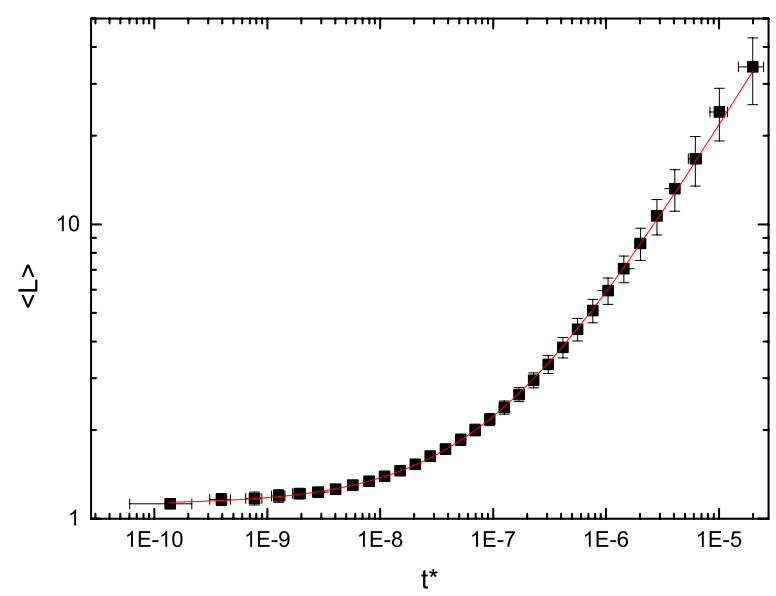

FIG. 6. The mean cluster size, $\langle L\rangle$, averaged over all 25 experimental runs, plotted as a function of the re-scaled time, $t^{*}$. The error bars correspond to the standard deviation of all the experimental runs. The solid line is a power law fit to the data of the form $\langle L\rangle=a+b t^{*^{z^{\prime}}}$ and is performed and plotted through these points in red. The fitting parameters are $a=1.12, b=3.1 \cdot 10^{4}$, and $z^{\prime}=0.61$. 
be close enough to be considered as neighbours. Consistent with this, we find that $a=1.12$. The pre-factor is found to be $b=3.1 \cdot 10^{4}$ and the exponent $z^{\prime}=0.61$. The value of $z^{\prime}$ is in very good agreement with the deterministic model, Eq. (11), but also lies in the range of diffusive theory, Eq. (5), which prevents any distinction using the exponent. Despite the fact that we cannot distinguish mechanisms from the exponent $z^{\prime}$, we can do so from the exponents characterising the packing fraction and magnetic field dependence, which indicate a deterministic mechanism. Importantly, our approach allows the complete characterisation of the field and packing fraction dependence of chain formation without pre-assuming a mechanism. Combining the exponents $\alpha$ and $\beta$ and the fitting parameters $a, b$, and $z^{\prime}$, thus fully predicts the time at which a given mean cluster size is reached.

\section{CONCLUSIONS}

The magnetic field induced aggregation of superparamagnetic colloidal particles into linear chains has been studied in two dimensions using video-microscopy. We find that the mean cluster size grows as a power law in time. The exponents that characterise the packing fraction and magnetic field dependence of the mean cluster size are directly determined from the experimental data and lead to an excellent data collapse upon plotting the mean cluster size as a function of the rescaled time. Comparison to diffusion limited and deterministic models for chain formation shows that the aggregation kinetics in our system is consistent with a deterministic mechanism and that the contribution of diffusion is thus minimal.

\section{ACKNOWLEDGMENTS}

We thank William Sampson, David Robinson, and Mark Wilson for useful discussions and acknowledge EPSRC (No. $\mathrm{EP} / \mathrm{J} 001902 / 1$ ) for financial support.

${ }^{1}$ S. Fraden, A. J. Hurd, and R. B. Meyer, Phys. Rev. Lett. 63, 2373 (1989).

${ }^{2}$ A. T. Skjeltorp, Phys. Rev. Lett. 51, 2306 (1983).

${ }^{3}$ J. H. E. Promislow, A. P. Gast, and M. Fermigier, J. Chem. Phys. 102, 5492 (1995).

${ }^{4}$ M. Fermigier and A. P. Gast, J. Colloid Interface Sci. 154, 522 (1992).

${ }^{5}$ L. He, M. Wang, J. Ge, and Y. Yin, Acc. Chem. Res. 45, 1431 (2012).

${ }^{6}$ R. G. Larson, The Structure and Rheology of Complex Fluids (Oxford University Press, New York, 1999).

${ }^{7}$ I. Bica, Y. D. Liu, and H. J. Choi, J. Ind. Eng. Chem. 19, 394 (2013).
${ }^{8}$ J. de Vicente, D. J. Klingenberg, and R. Hidalgo-Alvarez, Soft Matter 7, 3701 (2011).

${ }^{9}$ F. Donado, U. Sandoval, and J. L. Carrillo, Phys. Rev. E 79, 011406 (2009).

${ }^{10}$ J. Černák, G. Helgesen, and A. T. Skjeltorp, Phys. Rev. E 70, 031504 (2004).

${ }^{11}$ J. Černák and G. Helgesen, Phys. Rev. E 78, 061401 (2008).

${ }^{12}$ R. M. Erb, M. D. Krebs, E. Alsberg, B. Samanta, V. M. Rotello, and B. B. Yellen, Phys. Rev. E 80, 051402 (2009).

${ }^{13}$ G. Pál, F. Kun, I. Varga, D. Sohler, and G. Sun, Phys. Rev. E 83, 061504 (2011).

${ }^{14}$ F. Martínez-Pedrero, A. El-Harrak, J. C. Fernández-Toledano, M. TiradoMiranda, J. Baudry, A. Schmitt, J. Bibette, and J. Callejas-Fernández, Phys. Rev. E 78, 011403 (2008).

${ }^{15}$ F. Martínez-Pedrero, M. Tirado-Miranda, A. Schmitt, and J. CallejasFernández, Phys. Rev. E 76, 011405 (2007).

${ }^{16}$ R. E. Moctezuma, F. Donado, and J. L. Arauz-Lara, Phys. Rev. E 88, 032305 (2013).

${ }^{17}$ P. Domínguez-García, S. Melle, J. Pastor, and M. Rubio, Phys. Rev. E 76, 051403 (2007).

${ }^{18}$ P. Domínguez-García, J. M. Pastor, and M. A. Rubio, Eur. Phys. J. E 34, 36 (2011).

${ }^{19}$ G. Helgesen, A. T. Skjeltorp, P. M. Mors, R. Botet, and R. Jullien, Phys. Rev. Lett. 61, 1736 (1988).

${ }^{20}$ T. Ukai, H. Morimoto, and T. Maekawa, Phys. Rev. E 83, 061406 (2011).

${ }^{21}$ S. Melle, O. G. Calderón, M. A. Rubio, and G. G. Fuller, Phys. Rev. E 68, 041503 (2003).

${ }^{22}$ M. Hagenbüchle and J. Liu, Appl. Opt. 36, 7664 (1997).

${ }^{23}$ J. J. M. Janssen, J. J. M. Baltussen, A. P. van Gelder, and J. A. A. J. Perenboom, J. Phys. D: Appl. Phys. 23, 1447 (1990).

${ }^{24} \mathrm{~F}$. Martínez Pedrero, M. Tirado Miranda, A. Schmitt, and J. Callejas Fernández, J. Chem. Phys. 125, 084706 (2006).

${ }^{25}$ S. Melle, M. A. Rubio, and G. G. Fuller, Phys. Rev. Lett. 87, 115501 (2001).

${ }^{26}$ J. E. Martin, J. Odinek, and T. C. Halsey, Phys. Rev. Lett. 69, 1524 (1992).

${ }^{27}$ J. E. Martin and J. Odinek, J. Non-Cryst. Solids 172, 1135 (1994).

${ }^{28}$ J. M. Laskar, J. Philip, and B. Raj, Phys. Rev. E 82, 021402 (2010).

${ }^{29}$ D. Sohn, J. Magn. Magn. Mater. 173, 305 (1997).

${ }^{30}$ E. Climent, M. R. Maxey, and G. E. Karniadakis, Langmuir 20, 507 (2004).

${ }^{31}$ D. Liu, M. R. Maxey, and G. E. Karniadakis, J. Micromech. Microeng. 15, 2298 (2005).

${ }^{32}$ G. Bossis, L. Iskakova, V. Kostenko, and A. Zubarev, Physica A 390, 2655 (2011).

${ }^{33}$ G. Bossis, P. Lançon, A. Meunier, L. Iskakova, V. Kostenko, and A. Zubarev, Physica A 392, 1567 (2013).

${ }^{34}$ E. K. O. Hellén, T. P. Simula, and M. J. Alava, Phys. Rev. E 62, 4752 (2000).

${ }^{35}$ M. Kolb, Phys. Rev. Lett. 53, 1653 (1984).

${ }^{36}$ S. Miyazima, P. Meakin, and F. Family, Phys. Rev. A 36, 1421 (1987).

${ }^{37}$ R. Botet, R. Jullien, and M. Kolb, J. Phys. A: Math. Gen. 17, L75 (1984).

${ }^{38}$ T. Vicsek and F. Family, Phys. Rev. Lett. 52, 1669 (1984).

${ }^{39}$ M. Miguel and R. Pastor-Satorras, Phys. Rev. E 59, 826 (1999).

${ }^{40}$ H. See and M. Doi, J. Phys. Soc. Jpn. 60, 2778 (1991).

${ }^{41}$ J. Faraudo, J. S. Andreu, and J. Camacho, Soft Matter 9, 6654 (2013).

${ }^{42}$ J. S. Andreu, J. Camacho, and J. Faraudo, Soft Matter 7, 2336 (2011).

${ }^{43}$ P. Meakin, J. Colloid Interface Sci. 102, 491 (1984).

${ }^{44}$ A. E. Koser, N. C. Keim, and P. E. Arratia, Phys. Rev. E 88, 062304 (2013).

${ }^{45}$ J. González-Gutiérrez, J. L. Carrillo-Estrada, and J. C. Ruiz-Suárez, Phys. Rev. E 89, 052205 (2014).

${ }^{46}$ B. A. Wael, A. Malek, and M. Ali, J. Appl. Phys. 112, 094910 (2012). 